\section{Abnormal mucosa in gastroenteritis}

and RUDGE TOWNLEY

Department of Gastroenterology, Royal Children's Hospital,

Parkville 3052,

Melbourne,

Sir,

We thank Drs. Walker-Smith and Rossiter (1973) for their comments regarding abnormal mucosa in gastroenteritis, but feel constrained to reply to one or two points at this late stage.

They reject the statement that single biopsy specimens provide reliable information about the degree of damage to duodenal mucosa on the basis that 3 cases biopsied serially showing 'a consistent trend towards normality' is insufficient evidence. This may be a valid point and we did point out in the article (Barnes and Townley, 1973) that 'multiple biopsies ... . would be required to exclude the possibility of patchy damage in the duodenum'. Walker-Smith's (1972) postmortem studies of small bowel from children with abnormal mucosa refer only to gross morphology. The grading of mucosal damage in our study included degree of infiltration of the lamina propria and epithelial damage, which is probably more relevant when disaccharidase depression is being considered.

Lack of correlation between severity of biopsy abnormality and clinical status may not be related only to incorrect histological sampling, as Walker-Smith and Rossiter suggest. Surely biochemical changes are likely to be more relevant in a situation where fluid and electrolyte loss is the prime mechanism causing symptoms?

If the patchy nature of the lesion is the cause of lack of correlation of persisting sugar intolerance with disaccharidase depression, why is the sugar intolerance almost completely confined to the infants under 6 months of age? Histological damage was no more severe in this group. One would not expect biopsies to sample areas of lesser damage in the group under 6 months and areas of greater damage in the 6- to 12-month group.

It should be mentioned that approximately $75 \%$ of patients had Clinitest-positive stools $\left(>\frac{1}{2} \%\right)$ in the first 48 hours of the illness. The article was referring, however, to persisting sugar intolerance lasting more than 1 week.

While accepting some of the points made by your correspondents as worthy of consideration, we feel that their own explanations do not satisfactorily answer the questions posed by our study.

GRAEME BARNES

Department of Paediatrics and Child Health, Medical School, University of Otago, Dunedin, New Zealand, 